\title{
Stiffness Matrices of Isoparametric Four-node Finite Elements by Exact Analytical Integration
}

\author{
Gautam Dasgupta, Member ASCE \\ Columbia University, New York, NY
}

\section{Key words:}

$\mathrm{C}^{++}$code, convex quadrilateral element, divergence theorem, exact integration, FORTRAN code, isoparametric shape functions, Taig isoparametric mapping, triangle with a side node, Wachspress irrational expression.

\begin{abstract}
Explicit algebraic expressions needed to compute element stiffness matrices using procedural (FORTRAN) and object oriented $\left(\mathrm{C}^{++}\right)$computer programs are presented. Numerical illustrations for a convex quadrilateral and a triangle with a side node are included. The wide controversy due to conventional element level approximate numerical quadrature within the computational square domain, in $\eta$ and $\xi$ coordinates, is completely resolved here by the closed form analytical integration within the physical element, in $x$ and $y$ coordinates.
\end{abstract}

May 30, 2006 


\section{Introduction}

The finite element basis functions, $\Phi$, are so chosen that their linear combination $u$ will faithfully approximate solutions, within a domain $\Omega$ with boundary $\Gamma$, for field equations (subjected to a forcing function $f$ ) of mathematical physics (with appropriate boundary conditions $g$ ):

$$
\begin{aligned}
\mathcal{L} u & =f ; \quad \text { where } \mathcal{L} \text { is an elliptic spatial operator in } \Omega \\
\mathcal{B} u & =g ; \quad \text { where } \mathcal{B} \text { is the boundary operator on } \Gamma \\
u & =\sum_{i} a_{i} \Phi_{i}
\end{aligned}
$$

In the light of the Ritz pioneering formulation, ref. [6], the constants $a_{i}$ are determined by minimizing an energy type scalar. These norms are well known for the Laplacian, and elastostatic and vibration problems, ref. [2].

In order to cover an arbitrary geometrical domain with tiles (i.e., their one, two and three-dimensional analogs) $\Omega_{i}$, finite element shape functions $\phi$ are constructed as linear combinations of the bases:

$$
\begin{aligned}
\phi_{i}=\sum_{j} \alpha_{i j} \Phi_{j} \quad & \text { with local support: } \\
& \phi_{i}=0 \quad \text { outside } \Omega_{i}, \quad \alpha_{i j} \text { are constants }
\end{aligned}
$$

The values at each element's node constitute the discrete representation of the continuum solution $u$ of equation (1). These nodal responses are obtained via the constants $\alpha_{i j}$ that are determined by employing a suitable integral norm, e.g., the potential energy in linear elastostatic problems.

Here the focus is on linear boundary value problems where the following local conditions in terms of the stress and strain tensors, $\sigma$ and $\epsilon$, respectively, 
prevail:

$$
\begin{aligned}
\sigma_{i j, j} & =f_{i}: \text { equilibrium statement } \\
\sigma_{i j} & =C_{i j k l} \epsilon_{k l}: \text { constitutive relation }
\end{aligned}
$$

Standard indicial notations, which can be found in text books e.g., ref. [7], are used through out this paper.

The nodal values describe displacement fields within an element via shape functions (refer to any standard text — ref. $[1,5,13]$ — on finite elements for details). The continuum strain, $\epsilon_{i j}$ and nodal discrete displacement variables, $\left\{u_{i}\right\}$, at the element level are related by a matrix relation in which the strain tensor $\epsilon_{i j}$ is represented in terms of a one-dimensional list $\varepsilon_{k}$ corresponding to the stresses $\sigma_{m}$ :

$$
\sigma=\left\{\begin{array}{c}
\sigma_{x x}=\sigma_{x} \\
\sigma_{y y}=\sigma_{y} \\
\sigma_{x y}=\tau
\end{array}\right\} ; \quad \varepsilon=\left\{\begin{array}{c}
\epsilon_{x x}=\epsilon_{x} \\
\epsilon_{y y}=\epsilon_{y} \\
2 \epsilon_{x y}=\gamma
\end{array}\right\} ; \quad\left\{\varepsilon_{i}\right\}=\left[b_{i j}\right]\left\{u_{j}\right\}
$$

where $\left[b_{i j}\right]$ is the strain-displacement matrix.

The constitutive relation (for the plane stress and plane strain situations) in the matrix form:

$$
\left\{\begin{array}{c}
\sigma_{x} \\
\sigma_{y} \\
\tau
\end{array}\right\}=[d]\left\{\begin{array}{c}
\varepsilon_{x} \\
\varepsilon_{y} \\
\gamma
\end{array}\right\}
$$

is then invoked to obtain the element stiffness matrix $k$ as the following integral:

$$
\begin{aligned}
{[k] } & =\int_{\Omega}[\vartheta(x, y)] \mathrm{d} x \mathrm{~d} y ; \quad \vartheta: \text { energy density } \\
& =\int_{\Omega}[b(x, y)]^{T}[d(x, y)][b(x, y)] \mathrm{d} x \mathrm{~d} y
\end{aligned}
$$


For (convex) quadrilaterals, conventionally in almost all finite element commercial programs, the aforementioned relations, equation (5) and equation (7), in the physical $(x, y)$ coordinates, as in Figure 1, are transformed on a computational square, Figure 2, described in $(\eta, \xi)$ variables.

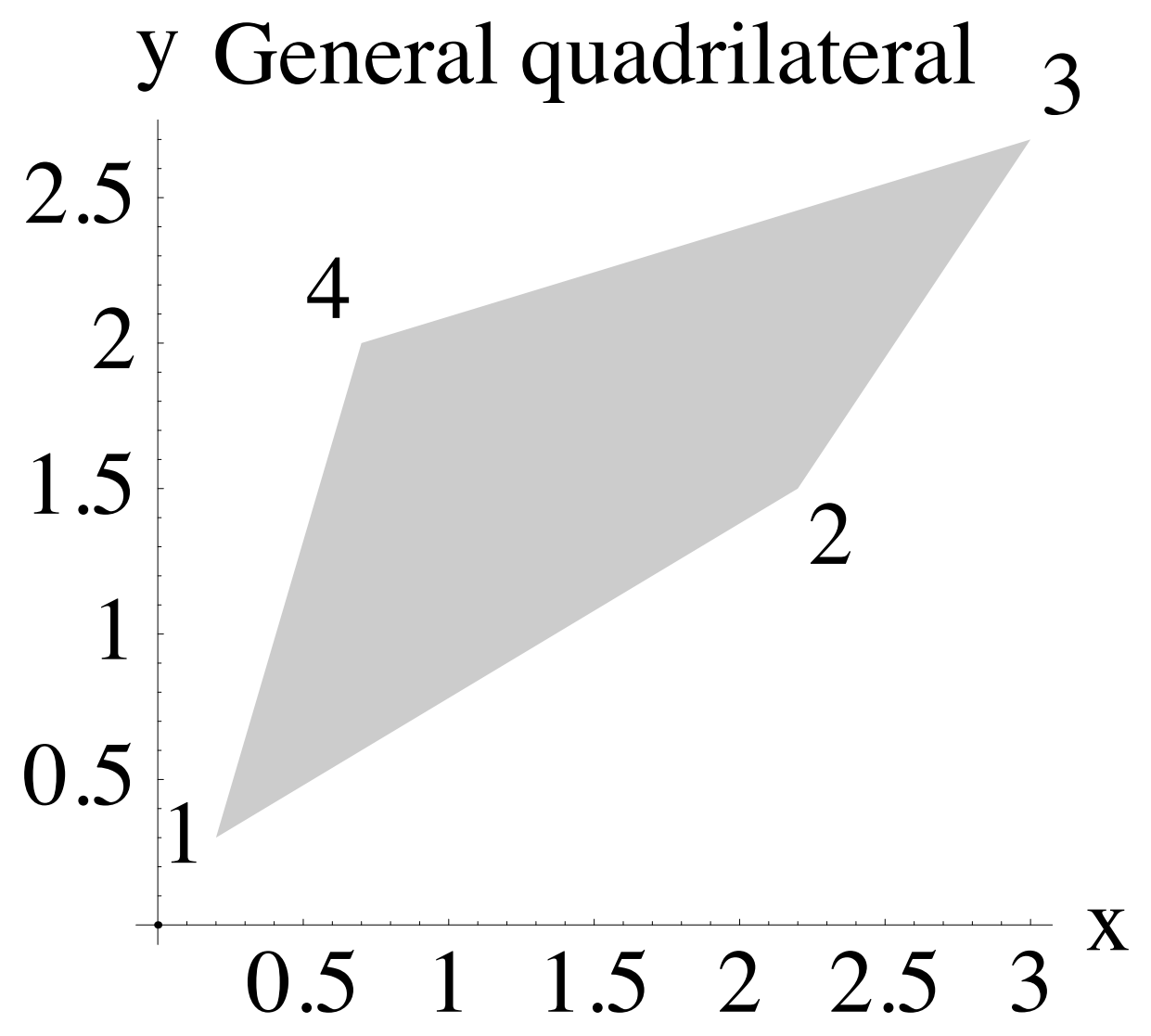

Figure 1: A quadrilateral element in the physical $(x, y)$ domain 


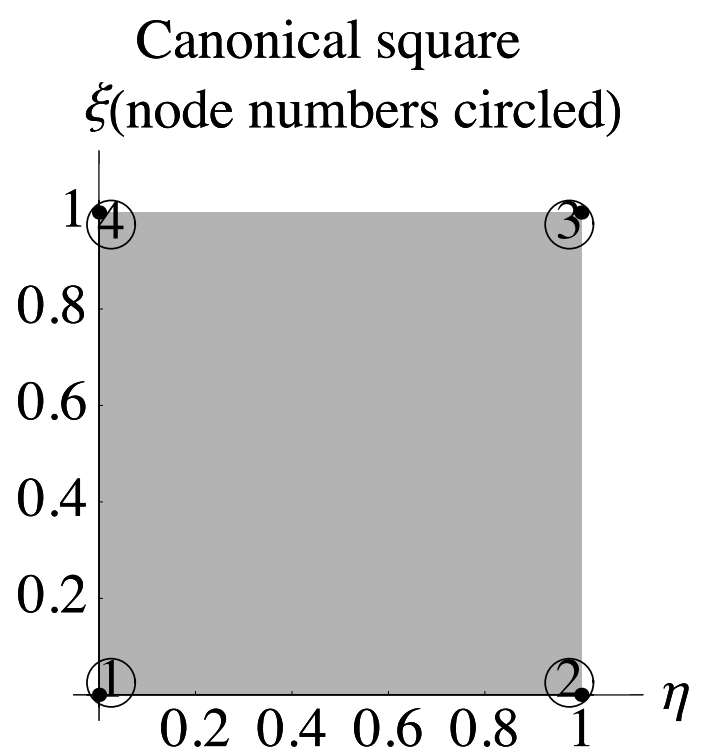

Figure 2: The canonical element $\Omega^{\circ}$ in the computational $(\eta, \xi)$ domain

The isoparametric formulation, which uses shape functions in the canonical region $\Omega^{\circ}$ (in terms of the $\eta$ and $\xi$ variables) have led successful evaluation of element stiffness matrices:

$$
[k]=\int_{\Omega}[\vartheta(x, y)] \mathrm{d} x \mathrm{~d} y=\int_{\Omega^{o}}[\vartheta(\eta, \xi)]\|J\| \mathrm{d} \eta \mathrm{d} \xi
$$

where: $[J]=\frac{\partial(x, y)}{\partial(\eta, \xi)}$ : the Jacobian matrix

and: $\|J\|$ is the determinant of $[J]$

where the strain energy density matrix element $\vartheta_{i j}(x, y)$ stands for $b_{k i}(x, y) d_{k l} b_{l j}(x, y)$, with implied summation convention.

In the available finite element literature, on the (computational) square domain $\Omega^{\circ}$ a suitable Gaussian quadrature is carried out to evaluate the 
entries of the stiffness matrix from equations (8), (9) and (10). The numerical value of the integrant is computed at a pre-determined number of isolated Gauss points, hence, the degree of accuracy of such quadratures becomes controversial, vide ref. [8].

The objective of this paper is to carry out the exact (close form analytical) integration (rather than the quadrature) in the physical $(x, y)$ domain, as stated in equation (7).

\section{Analytical integration of energy density}

In order to avoid any numerical error in generating stiffness matrices for two-dimensional (plane) elements a symbolic program in ref. [3] evaluates:

$$
\int_{\Omega} f(x, y) \mathrm{d} x \mathrm{~d} y ; \text { for an arbitrary } f
$$

in closed algebraic form within arbitrary polygons. The area integral is converted into the one along the perimeter by using the divergence theorem.

To compute the numerical value of the integral in equation (11) on $\Omega_{i}$, which is a (closed) polygon (irrespective of whether it is convex or concave), one needs a closed form expression for the indefinite integral:

$$
\mathcal{F}_{x}=\int f(x, y) \mathrm{d} x \text { or } \mathcal{F}_{y}=\int f(x, y) \mathrm{d} y
$$

The development and the associated proof appear in ref. [3] in detail. For convenience, only the $x$-integral $\mathcal{F}_{x}$, is used in this paper. 


\subsection{Stiffness matrix}

The stiffness matrix $[k]$ from the strain-displacement and constitutive rela-

tions, $[b]$ and $[d]$ is given in equation (7). Due to the algebraic structure of a typical isoparametric shape function (a linear term in $x$ and $y$ plus the square root of a quadratic in $x$ and $y$ ), a generic term in $[b]$ becomes:

$$
\text { a constant }+\frac{\partial}{\partial x} \text { or } \frac{\partial}{\partial y} \text { of } \sqrt{\text { a quadratic in } x \text { and } y}
$$

To facilitate the development of FORTRAN or C codes, this paper assumes a constant material property throughout the element (i.e., $[d]$ : constant). For users of a computer algebra system any constitutive inhomogeneity (to depict a variable property) can be incorporated in the symbolic code.

\section{Benchmark examples}

\subsection{A quadrilateral with vertex nodes}

As in Figure 1, with the nodes at:

$$
\left(\frac{1}{5}, \frac{3}{10}\right),\left(\frac{11}{5}, \frac{3}{2}\right),\left(3, \frac{27}{10}\right),\left(\frac{7}{10}, 2\right)
$$


the isoparametric shape functions (from ref. [4]) are:

$$
\begin{aligned}
& \phi_{1}=-\frac{9187}{5168}+\frac{2705 x}{2584}-\frac{3545 y}{2584}+\frac{55 r}{5168} \\
& \phi_{2}=-\frac{24521}{5168}-\frac{3555 x}{2584}+\frac{3035 y}{2584}-\frac{89 r}{5168} \\
& \phi_{3}=-\frac{9781}{2584}+\frac{2015 x}{1292}-\frac{1375 y}{1292}+\frac{35 r}{2584} \\
& \phi_{4}=\frac{2349}{1292}-\frac{795 x}{646}+\frac{815 y}{646}-\frac{9 r}{1292} \\
& r=\sqrt{2500 x^{2}+3000 x y-66380 x+900 y^{2}+42860 y+78457}
\end{aligned}
$$

Generic forms related to the square root of a quadratic expression are:

$$
r=\sqrt{1+a_{1} x+a_{2} x^{2}+a_{3} y+a_{4} y x+a_{5} y^{2}} ; \quad \frac{\partial r}{\partial x}=r_{, x} ; \quad \frac{\partial r}{\partial y}=r_{, y}
$$

The plane stress constitutive matrix is:

$$
[d]=\frac{1}{15}\left[\begin{array}{ccc}
16 & 4 & 0 \\
4 & 16 & 0 \\
0 & 0 & 6
\end{array}\right] ; \quad \text { for } E=1, \nu=\frac{1}{4}
$$

To compute the element stiffness matrix the algebraic expressions for $\left[b_{i j}\right]$ in equation (5) are determined from equation (15) using the notation in equation (16). The three rows of the strain-displacement transformation 
matrix [b], when multiplied by a factor 5168 becomes:

the first row:

$55 r_{, x}+5410,0,-89 r_{, x}-7110,0,70 r_{, x}+8060,0,-36 r_{, x}-6360,0$

the second row:

$0,55 r_{, y}-7090,0,6070-89 r_{, y}, 0,70 r_{, y}-5500,0,6520-36 r_{, y}$

the third row:

$$
\begin{aligned}
& 55 r_{, y}-7090,55 r_{, x}+5410,6070-89 r_{, y},-89 r_{, x}-7110, \\
& 70 r_{, y}-5500,70 r_{, x}+8060,6520-36 r_{, y},-36 r_{, x}-6360
\end{aligned}
$$

Standard finite element text books, ref. [1, 5, 13], contain the full detail of computing $[b]$ and $[d]$ as indicated in equations (17) and (18).

The integrands encountered in the computation of the element stiffness matrix $[k]=\int[b]^{T}[d][b] d \Omega$ are linear combinations of the following terms whose exact values, vide ref. [3], are:

$$
\begin{aligned}
\int_{\Omega} r_{, x} d \Omega & =-\frac{1347}{5} \\
\int_{\Omega} r_{, x}^{2} d \Omega & =\frac{625108}{21} \\
\int_{\Omega} r_{, y} d \Omega & =\frac{1259}{5} \\
\int_{\Omega} r_{, y}^{2} d \Omega & =\frac{106381}{4} \\
\int_{\Omega} r_{, x} r_{, y} d \Omega & =-\frac{306642}{11}
\end{aligned}
$$

These values are used to compute all terms in the element stiffness matrix 
leading to:

$$
[k]=\left[\begin{array}{cccccccc}
\frac{1172}{4481} & -\frac{49}{8073} & -\frac{4747}{24413} & -\frac{153}{9601} & \frac{744}{10985} & -\frac{838}{6905} & -\frac{3269}{24245} & \frac{643}{4485} \\
-\frac{49}{8073} & \frac{5073}{11731} & \frac{892}{17583} & \frac{753}{7187} & -\frac{838}{6905} & -\frac{38}{2135} & \frac{344}{4485} & -\frac{6460}{12437} \\
-\frac{4747}{24413} & \frac{892}{17583} & \frac{10795}{8257} & -\frac{7738}{13009} & -\frac{2389}{7060} & \frac{2290}{14729} & -\frac{7613}{9829} & \frac{3672}{9449} \\
-\frac{153}{9601} & \frac{753}{7187} & -\frac{7738}{13009} & \frac{13427}{8694} & \frac{1852}{8337} & -\frac{4874}{6283} & \frac{3672}{9449} & -\frac{8467}{9694} \\
\frac{744}{10985} & -\frac{838}{6905} & -\frac{2389}{7060} & \frac{1852}{8337} & \frac{25473}{72500} & -\frac{232}{5683} & -\frac{859}{10645} & -\frac{479}{7989} \\
-\frac{838}{6905} & -\frac{38}{2135} & \frac{2290}{14729} & -\frac{4874}{6283} & -\frac{232}{5683} & \frac{5991}{10982} & \frac{62}{9241} & \frac{437}{1762} \\
-\frac{3269}{24245} & \frac{344}{4485} & -\frac{7613}{9829} & \frac{3672}{9449} & -\frac{859}{10645} & \frac{62}{9241} & \frac{11169}{11281} & -\frac{1898}{4021} \\
\frac{643}{4485} & -\frac{6460}{12437} & \frac{3672}{9449} & -\frac{8467}{9694} & -\frac{479}{7989} & \frac{437}{1762} & -\frac{1898}{4021} & \frac{15825}{13823}
\end{array}\right]
$$

It is always advisable to check the positive semi-definiteness of element stiffness matrices. The eigenvalues of $[k]$ in equation (20) are:

$$
\frac{75}{23}, \frac{41}{30}, \frac{59}{54}, \frac{17}{33}, \frac{10}{29}, 0,0,0
$$

Observe that three rigid body modes are indicated by zero eigenvalues and the remaining five are all positive.

\subsection{A triangle with a side node}

Figure 3 shows a triangle with a side node. The nodal coordinates are:

$$
(1,1),\left(\frac{8}{5}, \frac{13}{10}\right),(3,2),(2,3)
$$




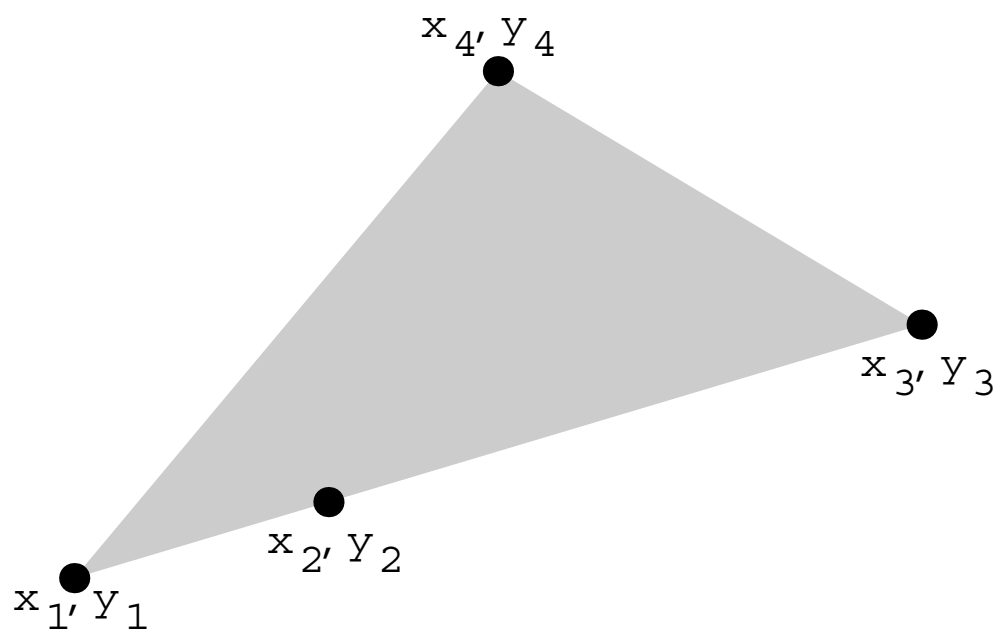

Figure 3: Triangle with a side node

The four isoparametric shape functions, vide ref. [4], are:

$$
\begin{aligned}
& \phi_{1}=\frac{16}{9}-\frac{7 x}{18}-\frac{8 y}{9}+\frac{r}{18} \\
& \phi_{2}=-\frac{10}{63}+\frac{5 x}{63}+\frac{50 y}{63}-\frac{5 r}{63} \\
& \phi_{3}=-\frac{2}{7}+\frac{9 x}{14}-\frac{4 y}{7}+\frac{r}{42} \\
& \phi_{4}=-\frac{1}{3}-\frac{x}{3}+\frac{2 y}{3}
\end{aligned}
$$

where: $r=\sqrt{169 x^{2}+104 y x-928 x+16 y^{2}+296 y+424}$

With the material properties $E=1, \nu=\frac{1}{4}$, the constitutive matrix $[d]$ under the plane stress condition has stated in equation (17).

The strain-displacement transformation matrix becomes:

$$
\frac{1}{126}\left[\begin{array}{cccccccc}
7 r_{, x}-49 & 0 & 10-10 r_{, x} & 0 & 3 r_{, x}+81 & 0 & -42 & 0 \\
0 & 7 r_{, y}-112 & 0 & 100-10 r_{, y} & 0 & 3 r_{, y}-72 & 0 & 84 \\
7 r_{, y}-112 & 7 r_{, x}-49 & 100-10 r_{, y} & 10-10 r_{, x} & 3 r_{, y}-72 & 3 r_{, x}+81 & 84 & -42
\end{array}\right]
$$


The following exact values:

$$
\begin{aligned}
\int_{\Omega} r_{, x} d \Omega & =-\frac{24}{5} \\
\int_{\Omega} r_{, x}^{2} d \Omega & =\frac{3031}{24} \\
\int_{\Omega} r_{, y} d \Omega & =\frac{138}{5} \\
\int_{\Omega} r_{, y}^{2} d \Omega & =\frac{6867}{10} \\
\int_{\Omega} r_{, x} r_{, y} d \Omega & =-\frac{6102}{31}
\end{aligned}
$$

are used to compute all terms in the following element stiffness matrix:

$$
[k]=\frac{1}{97020}\left[\begin{array}{cccccccc}
107800 & -29106 & -116424 & 48510 & -26460 & -1980 & 35280 & -17640 \\
-29106 & 90956 & 55440 & -124740 & -1980 & 8820 & -24255 & 24255 \\
-116424 & 55440 & 158760 & -66701 & 0 & -10780 & -43120 & 21560 \\
48510 & -124740 & -66701 & 218295 & -4042 & -17640 & 21560 & -75460 \\
-26460 & -1980 & 0 & -4042 & 60637 & -12127 & -35280 & 17640 \\
-1980 & 8820 & -10780 & -17640 & -12127 & 35280 & 24255 & -24255 \\
35280 & -24255 & -43120 & 21560 & -35280 & 24255 & 43120 & -21560 \\
-17640 & 24255 & 21560 & -75460 & 17640 & -24255 & -21560 & 75460
\end{array}\right]
$$

The eigenvalues of $[k]$ are:

$$
\frac{181}{43}, \frac{178}{41}, \frac{41}{38}, \frac{4}{7}, \frac{15}{37}, 0,0,0
$$




\section{Closed form algebraic expressions for procedural and objected-oriented computer programs}

In order to carry out the exact integrations that are essential to construct the stiffness matrix, the method outlined in ref. [3] can be utilized. To evaluate:

$$
\int_{\Omega} f(x, y) d \Omega
$$

by applying the divergence theorem, the following indefinite integral:

$$
\int f(x, y) d x
$$

is used. Specifically, the following four integrals:

$$
\int \frac{\partial r}{\partial y} d x, \int\left(\frac{\partial r}{\partial x}\right)^{2} d x, \int \frac{\partial r}{\partial x} \frac{\partial r}{\partial y} d x, \int\left(\frac{\partial r}{\partial y}\right)^{2} d x
$$

are obtained (from Mathematica):

$$
\begin{aligned}
\int \frac{\partial r}{\partial y} d x & =\frac{1}{4 a_{2}^{3 / 2}}\left(2 a_{4} \sqrt{a_{2} r}+r_{7} \log \left(\frac{r_{1}}{\sqrt{a_{2}}}+2 \sqrt{r}\right)\right) \\
\int\left(\frac{\partial r}{\partial x}\right)^{2} d x & =a_{2} x-\frac{r_{4}}{2} \sqrt{r_{2}} \\
\int\left(\frac{\partial r}{\partial x} \frac{\partial r}{\partial y}\right)^{2} d x & =\frac{1}{8 a_{2}}\left(2 a_{2} r_{5} r_{7}+a_{4}\left(4 x a_{2}-r_{6} r_{7}-2 r_{4} \sqrt{r_{2}}\right)\right) \\
\int\left(\frac{\partial r}{\partial y}\right)^{2} d x & =\frac{1}{8 a_{2}^{2} \sqrt{r_{2}}}\left(4 a_{2}^{2} r_{4} r_{5}^{2}\right. \\
& +2 a_{2} a_{4}\left(a_{4}\left(x \sqrt{r_{2}}-2\left(3 a_{5} y^{2}+2 a_{3} y+1\right) r_{4}\right)-2 a_{1} r_{4} r_{5}\right) \\
& \left.+a_{4}\left(2 a_{4} r_{4} r_{6}^{2}+\sqrt{r_{2}} r_{3} r_{8}\right)\right)
\end{aligned}
$$


where:

$$
\begin{aligned}
& r_{1}=a_{1}+2 x a_{2}+y a_{4} \\
& r_{2}=-a_{1}^{2}-2 y a_{4} a_{1}-y^{2} a_{4}^{2}+4 a_{2}\left(a_{5} y^{2}+a_{3} y+1\right) \\
& r_{3}=2 a_{2}\left(a_{3}+2 y a_{5}\right)-a_{4}\left(a_{1}+y a_{4}\right) \\
& r_{4}=\tan ^{-1}\left(\frac{r_{1}}{\sqrt{r_{2}}}\right) \\
& r_{5}=a_{3}+2 a_{5} y \\
& r_{6}=a_{1}+a_{4} y \\
& r_{7}=2 a_{2} r_{5}-a_{4} r_{6} \quad \text { and } \\
& r_{8}=\log r
\end{aligned}
$$

All mathematical statements from equation (35) through (46) can be easily coded in any procedural, e.g., C and FORTRAN, or object-oriented programing language, e.g., $\mathrm{C}^{++}$and Java.

\section{Conclusions}

Closed form analytical integration of the strain energy density function, which is based on the explicit shape function expressions derived from the isoparametric formulation, alleviates the controversy, ref. [8], surrounding the order, precision and accuracy of numerical quadrature formulæ conjectured by researchers since the inception, vide ref. [9], of the versatile quadrilateral elements (and their three-dimensional analogs).

Existing text books and research publications, almost all of them, address 
different schemes of spatial integration on the computational domain in $\eta$ and $\xi$ on a unit square $\left(\Omega^{o}\right)$. Currently available symbolic computation programs can furnish expressions necessary for ready coding in a numerically efficient language (e.g., C, FORTRAN or $\mathrm{C}^{++}$) to generate stiffness matrices for the other kind of elements, notably, three-dimensional solids, thick plates and shells, for which the isoparametric formulation is indispensable.

The general form of shape functions in $x$ and $y$ coordinates contain square root terms where as those in the $\eta$ and $\xi$ system are strictly bilinear. This observation provides a conceptual link to connect the intuitive isoparametric scheme with the Wachspress irrational shape functions, ref. [10, 11]. Furthermore, these radical subexpressions indicate that there cannot be a clear designation about the algebraic degree of interpolants since a Taylor expansion will contain all higher power $x-$ and $y-$ terms. However, combinations of linear terms and square roots of quadratics indicate a consistency of having dimensionality that is amenable to first order representation.

The detailed steps of generating algebraic isoparametric shape functions in $x-$ and $y$ - variables is addressed by the author in ref. [4]. Computer algebra steps in the Mathematica programming language, ref. [12], is included in the Appendix. Using those subexpressions in a numeric (C, FORTRAN, $\mathrm{C}^{++}$) code the time efficiency can be considerably enhanced to generate isoparametric stiffness matrices for convex quadrilaterals and four-node triangles. 


\section{Acknowledgement}

The research was supported by the following grants from the National Science Foundation: "Concave Finite Element Shape Functions" CMS-0202232, "Workshop for Scientists and Engineers on Structural Deformations at the Historic Site of Angkor, in Cambodia" OISE-0456406, and "US-France Cooperative Research: Engineering Shape Calculation for Surgery, Biology and Anthropology" INT-0233570.

\section{Dedication}

This paper is dedicated to the memory of the late Professor Prasanta K. Sinha, who led an exemplary academic life and retired as the Chair of the Aerospace Engineering Department, Indian Institute of Technology, Kharagpur, India. He achieved the monumental task of initiating a prestigious conference to bring all branches of mechanics together in ICTACEM (International Conference of Theoretical, Applied, Computational and Experimental Mechanics). 


\section{References}

[1] Robert D. Cook. Finite Element Modeling for Stress Analysis. John Wiley, 1995.

[2] R. Courant. Variational methods for the solution of problems of equilibrium and vibration. Bulletin of the American Mathematical Society, 49:1-29, 1943.

[3] G. Dasgupta. Integration within polygonal finite elements. Journal of Aerospace Engineering, ASCE, 16(1):9-18, January 2003.

[4] G. Dasgupta. Closed form isoparametric shape functions of four-node convex finite elements. Journal of Aerospace Engineering, ASCE, 2006. accepted for publication.

[5] T J. R. Hughes. The Finite Element Method. Prentice-Hall, Inc., 1987.

[6] W. Ritz. Über eine neue methode zur lösung gewisser variationalprobleme der mathematischen physik. Journal Reine Angew. Math., 135, 1908.

[7] A. J. M. Spencer. Continuum Mechanics. Longman, 1980.

[8] G. Strang and G. J. Fix. An Analysis of the Finite Element Method. Prentice-Hall, Inc., Englewood Cliffs, N. J., 1973.

[9] I. C. Taig. Structural analysis by the matrix displacement method. Report S017, English Electric Aviation Report, England, 1961. 
[10] E. L. Wachspress. A Rational Basis for Function Approximation, volume 228 of Lecture Notes in Mathematics. Springer Verlag, 1971.

[11] E. L. Wachspress. A rational finite element basis. Academic Press, 1975.

[12] S. Wolfram. The Mathematica Book, System 5. Wolfram Media, NY, 2003.

[13] O. C. Zienkiewicz and R. L. Taylor. The Finite Element Method, volume 2-Solid Mechanics. Elsevier, New York, NY, 5th edition, 2000. 